Revue de phénoménologie

$27 \mid 2019$

Patočka

\title{
Le sacrifice du sujet chez Jan Patočka. Vers une ontologie réaliste.
}

Pierre Souq

\section{(2) OpenEdition}

1 Journals

Édition électronique

URL : https://journals.openedition.org/alter/1883

DOI : 10.4000/alter.1883

ISSN : 2558-7927

Éditeur :

Association ALTER, Archives Husserl (CNRS-UMR 8547)

Édition imprimée

Date de publication : 1 novembre 2019

Pagination : 83-99

ISBN : 978-2-9550449-5-7

ISSN : 1249-8947

\section{Référence électronique}

Pierre Souq, « Le sacrifice du sujet chez Jan Patočka. Vers une ontologie réaliste. », Alter [En ligne], 27 | 2019, mis en ligne le 22 décembre 2020, consulté le 13 juin 2021. URL : http://

journals.openedition.org/alter/1883 ; DOI : https://doi.org/10.4000/alter.1883

Ce document a été généré automatiquement le 13 juin 2021

Revue Alter 


\title{
Le sacrifice du sujet chez Jan Patočka. Vers une ontologie réaliste.
}

\author{
Pierre Souq
}

Où est le péril dans tout cela?

Jan Patočka

1 Alors qu'Edmund Husserl et Martin Heidegger sont largement commentés en France depuis près d'un siècle, le philosophe tchèque Jan Patočka, qui pourtant rassemble leurs pensées, est seulement traduit à partir des années $1980^{1}$. Sa pensée, qui peut paraitre moins abstraite, ramène le lecteur à quelque chose de plus réaliste, de plus proche de l'homme ordinaire, de plus facile à comprendre. Cependant, ce qui légitime sa présence aujourd'hui, c'est peut-être son caractère visionnaire, trop en avance pour son temps, plus moderne dans l'écriture car plus proche des problématiques que nous, français, traversons. En effet, s'il existe un moment français qui apparaît dans l'histoire de la phénoménologie au début des années 1980, il critique, comme Patočka, non seulement la possibilité de décrire un phénomène pur, mais aussi la réduction du sujet comme seul étant capable de le constituer et de lui donner sens². En rapport à la pensée de Patočka, Françoise Dastur souligne ainsi l'interprétation d'Émilie Tardivel qui consiste à parler de : "définition "historiale" de la liberté ou de "phénoménologie événementiale", dans des termes qui ne sont pas ceux employés par Patočka lui-même. 3 »

2 En effet, si Patočka semble abandonner le projet d'une « histoire universelle » dans les années 1930 au profit d'une phénoménologie, il est possible de voir, plus tard, non pas un retour à ses ambitions premières, mais la découverte d'une histoire vécue en-deçà du problème de la conscience. La difficulté qui est passée d'une conception du sujet transcendantal (avec Husserl) à une réflexion ontologique en rapport à la facticité de l'existence (avec Heidegger), se retrouve alors dans une philosophie historique ultérieure qui semble faire disparaître le sujet, puisqu'elle se dit «a-subjective ${ }^{4}$, tout en voulant maintenir un ton polémique où la liberté est à son fondement. Aussi, bien qu'il faille accepter que l'histoire se nourrisse de l'expérience du sujet, elle pourrait aussi en être la source. Dans ce sens, la force des événements au sein de la 
phénoménologie traditionnelle a souvent été négligée, les effets de l'histoire dépassant largement l'ébranlement esseulé du sujet dont l'existence même peut sembler insignifiante par rapport au réel qui persiste. À ce titre, si le monde a un impact réel sur le sujet, il peut s'agir soit d'événements ponctuels, en rapport à un contexte ou une "situation" (die Situation mais aussi der Stand), soit d'une histoire plus profonde qui traverse l'existence du sujet selon des « conceptions du monde » (die Weltanschauungen) ${ }^{5}$ qui s'imposent à son insu. Dans le premier cas, Patočka parle de "crises» ou de " guerres » en référence, à une histoire assez formelle et chronologique ; dans le second cas, il s'agit de la temporalité même de l'existence du sujet qui repose sur un « ébranlement principiel $»^{6}$, dû à l'opposition des conceptions qui constituent le moteur de l'histoire. Aussi, si le sujet est ébranlé par les événements en raison de la nature conflictuelle de son existence, il faut mesurer sa capacité à réagir, c'est-à-dire à agir sans penser, suite à une expérience dont les causes sont vécues comme si elles étaient extérieures. Cette situation, déterminée a priori par des jeux de forces qui dépassent l'action individuelle, est immédiatement incomprise par le sujet qui la vit comme une " autoaliénation" pouvant mener à l'«abdication de soi $»^{7}$. Alors, ce que nous voulons montrer, c'est que, si la transformation du concept de "phénomène " en celui d'« événement » est bien comprise aujourd'hui d'un point de vue phénoménologique, elle manifeste une ontologie réaliste où le sujet peut disparaitre, considéré comme un étant passif et se laissant aller, subjugué par les événements du monde. Aussi, si le sujet reste passif «dans " son monde, c'est en raison à la fois d'une tendance naturelle à vivre de façon naïve et d'une prédisposition à "sacrifier » sa pensée devant l'autorité de conceptions qui lui paraissent surpuissantes. Ce sacrifice apparaît lorsque le sujet est submergé par les conceptions du monde qui, non seulement lui donne le sentiment que tout va mal, mais il est maintenu au niveau du monde ambiant, le flux de l'activité quotidienne entretenant la mise en retrait de la pensée du sujet. Il s'agira donc de comprendre la phénoménologie de Patočka, comme une ontologie réaliste qui fait de l'être même du sujet, non plus un oubli, comme chez Heidegger, mais comme un sacrifice involontaire, la tendance naturelle de l'homme étant de rester «dans » le monde, et non pas de le décrire ou de le comprendre. Cependant, nous verrons que ce sacrifice est ambivalent car, derrière la passivité ordinaire, le sujet peut réagir si les événements de l'histoire lui paraissent importants. Peut-être sera-t-il alors possible de comprendre le concept de sacrifice, non pas comme un problème théorique, mais comme l'expression même de l'existence de l'homme qui, devant la réalité du monde déclinant, réagit de façon concrète, mais passive, en oubliant au fond, ce qu'il est vraiment.

\section{La situation du monde et le sentiment de crise}

Dès sa thèse d'habilitation en 1936, Patočka emploie le terme de "situation " pour qualifier le rapport de l'être-au-monde dans l'histoire. Initiant un détachement vis-àvis de l'ego transcendantal husserlien, Patočka reprend à Heidegger l'idée qu'il existe une tonalité fondamentale qui sous-tend l'existence humaine. Dans ce sens, non seulement le sujet est pris dans le flux de l'activité ordinaire en raison d'une certaine disposition d'humeur (Befindligkeit), mais celle-ci est plus ou moins ouverte aux divers événements du monde, ce qui modifie son expression et les sentiments qu'il éprouve. Chez Patočka, le monde est donc ambivalent car il est à la fois la condition d'existence du sujet en tant que fond duquel surgit son histoire, mais aussi l'objet d'une étude 
descriptive, telle que la philosophie peut le comprendre, et qui donne lieu à des « conceptions du monde » :

Le monde ne peut rien voir de la philosophie si ce n'est sa projection mondaine; la philosophie, en revanche, voit le monde tel qu'il est effectivement - car c'est là son thème. Le philosophe est extérieurement sans défense contre le monde, le monde intérieurement sans défense contre la philosophie ${ }^{8}$.

4 À la suite de Husserl, Patočka montre que le " monde » est d'abord celui de tous les hommes, il est ordinaire et relève d'un esprit naïf, il est vécu de façon immédiate comme réel au sein duquel les actions s'accomplissent. Fonds du quotidien, le monde n'est pas problématique dans cette conception car il n'est jamais questionné, ce qui ne signifie pas que le sujet ne puisse pas réfléchir en son sein. Simplement, sa réflexion porte sur des choses de l'activité ordinaire, non sur les conditions phénoménologiques de son apparition. À l'inverse, la pensée du philosophe manifeste une prise de recul réflexive dont l'épochè est la méthode primordiale, son esprit se trouvant au-dessus de la vie de l'homme ordinaire qui ne comprend pas son origine et ses fondements. En effet, c'est dans la description du monde, que la philosophie s'affranchit du monde au sein duquel l'homme ordinaire est engagé sans y penser. À la différence de Husserl, qui fait de cette activité théorique une mise à distance pure de l'ego transcendantal, cet écart reste immanent chez Patočka, c'est-à-dire expérientiel, maintenu dans une forme spirituelle d'intériorité au sein de laquelle le sujet continue de vivre. Cette nouvelle forme d'intériorité, qui consiste à penser le monde primordial du sujet, manifeste toujours un intérêt dont le sens est réel car il est amené à comprendre concrètement le monde au sein duquel il vit. Cette tendance à questionner le monde, qui s'éloigne de la pensée d'un sujet pur comme chez Husserl, manifeste ce que Patočka appelle la "problématicité »", c'est-à-dire une réflexion sur la tendance même du sujet à vouloir saisir le monde de façon unitaire et totalisante. Ce questionnement, qui n'est pas idéal ou purement théorique chez Patočka, manifeste alors toujours une certaine empirie par rapport à l'existence humaine. L'expérience philosophique, dont la problématicité est le fond, permet de poser un regard a-subjectif sur le monde à partir d'une attitude de refus s'apparentant à l'épochè husserlienne, bien qu'elle s'insère dans le réel. C'est donc l'expérience du rejet et de la retenue dont la philosophie témoigne, laquelle permet d'ouvrir l'histoire à l'étude des conflits intérieurs de l'homme où l'attitude du philosophe consiste à sacrifier à la fois le monde ordinaire, puisqu'il en fait l'objet d'une pensée, mais aussi l'idéal d'une pensée pure, puisqu'elle reste engagée dans un monde. Cette mise entre parenthèses du monde ordinaire où apparait la "problématicité » du monde, prend le sens du sacrifice puisqu'elle donne le sentiment que celui qui l'opère «sort » du monde immanent au sein duquel il était plongé. Chez Patočka, il marque la retenue de la pensée qui tente une plus grande neutralité vis-à-vis de la thèse du monde, tout en comprenant la difficulté à prononcer un sens réellement objectif afin de comprendre ce qu'il est concrètement. La retenue de celui qui veut penser, n'est donc pas simplement une attitude théorique de l'esprit, comme le doute cartésien et l'épochè husserlienne le laissait entendre - elle engage l'existence de l'homme toute entière qui ne veut plus vivre ordinairement en raison du sentiment négatif de sa chute ou de son déclin ${ }^{10}$. Ainsi, ce n'est pas tant l'aspect problématique de la vie qui rend manifeste l'histoire sur un plan théorique, que l'apparition de son caractère problématique suite au vécu d'un sentiment de crise. 
5 Dans Platon et l'Europe, Patočka fait du naufrage en mer la métaphore de la vie, l'homme étant toujours engagé dans une situation douloureuse, devant faire face à des événements imprévus :

L'homme est toujours dans une situation désespérée [...] il est toujours un être engagé dans une aventure qui, en un sens, ne peut se terminer bien. [...] Notre aventure est vouée à l'échec en ce sens aussi que chaque individu est mortel ${ }^{11}$.

Il existe ainsi à la fois une " tonalité d'humeur $»^{12}$ qui permet à l'homme d'appréhender la vie de façon ordinaire, mais aussi la possibilité d'un sentiment occasionnel, lié à la réception d'événements extérieurs qui ébranlent l'existence du sujet à un moment " $t$ " d'une époque donnée, que la pensée du philosophe peut essayer de saisir en raison de son caractère de crise. Il faut alors d'abord distinguer la condition a priori naturelle de l'homme qui fait de lui l'acteur de son existence propre, de la production de la situation elle-même qui dépend d'une histoire dont les fondements paraissent a-subjectifs pour celui qui les pense. Si le "sentiment de crise», chez le sujet, est l'expression d'événements extérieurs qui entrent en résonance avec l'ébranlement naturel de son existence, il doit pouvoir comprendre son origine et ses fondements, à condition d'en apercevoir le caractère dramatique. Plus loin même, cette attitude de l'esprit ne doit pas être seule celle du philosophe, mais animer aussi l'esprit de l'homme ordinaire qui doit s'éveiller afin de vivre mieux. La phénoménologie, selon Patočka, comporte ainsi une essence profondément polémique car elle s'oppose aux conceptions du monde ambiant qui aliènent l'homme et le font abdiquer, mais aussi parce qu'au-delà, se trouve un projet politique de reconstruction de l'Europe à partir d'une réforme profonde des mentalités. C'est dans ce sens qu'il emprunte à Platon le concept de "souci de l'âme ", lequel incarne le combat réel de la pensée contre l'opinion et le dogme, tout comme le début de l'histoire, dans son opposition au mythe. Chez Platon, en effet, le «monde mythique » est l'équivalent du "monde naturel » chez Patočka, dans le sens où il marque le flux de la vie chez l'homme ordinaire qui ne pense pas de façon problématique ${ }^{13}$. Dans ce sens, l'opposition des Idées et des objets sensibles manifeste une séparation originelle, existentielle et "chorismatique ${ }^{14}$, dont la dialectique permet un réveil de l'esprit et un détachement du sol ordinaire pour élaborer l'histoire. Cette histoire, si elle marque la sortie du mythe, affirme le droit à la parole du sujet qui dans sa négation de la parole des Dieux peut constater lui-même l'aspect problématique du monde à partir du sentiment de son déclin :

Socrate et Platon sont des problématiseurs de la vie, des hommes qui n'acceptent pas la réalité telle qu'elle se donne, mais la voient ébranlées. Ébranlement qui les amène cependant à conclure à la possibilité d'une autre vie, d'une orientation différente de l'existence, d'un fondement nouveau qui donnerait alors seulement un critère de l'être et du non-être. Ils en sont si fermement convaincus qu'ils défient la réalité naïve en combat ${ }^{15}$.

7 Si Socrate et Platon s'opposent ainsi au monde au sein duquel ils vivent, c'est non seulement parce qu'ils ont le sentiment d'être ébranlés par les divers événements du monde, mais aussi parce qu'ils veulent faire acte politique en montrant la réalité telle qu'elle se trouve à l'homme ordinaire. Dans le projet de remonter aux Idées chez Platon se trouve bien une attitude épochale qui consiste non seulement à décrire le monde tel qu'il est, mais au fond, à faire sortir l'homme ordinaire de sa "prison » pour qu'il puisse voir la « lumière » (c'est-à-dire la vérité) et a fortiori éclairer lui-même la cité. Aussi, parce que les philosophes sont rares et peu nombreux, leur engagement comporte un risque, à la fois celui d'être incompris par le peuple, mais d'être 
condamné. Dès lors, l'engagement du philosophe qui a déjà sacrifié le monde commun en raison du sentiment que tout va mal, se retrouve dans une situation où il risque maintenant de perdre la vie, sous couvert qu'il veut partager ses idées aux autres ${ }^{16}$.

\section{Les conceptions du monde dans l'histoire}

8 Il est des auteurs qui se sacrifient toute leur vie et qui jamais n'exposent vraiment le mouvement de leur existence - celle de Patočka est complexe, teintée de phénoménologie, d'histoire, de pédagogie et de politique ${ }^{17}$. L'engagement de Patočka est total et le mènera à la même fin que Socrate. Aussi, si toute pensée paraît subjective, elle comporte des «conceptions du monde » qui dépendent de différentes époques et sont plus ou moins saillantes selon les situations vécues par le sujet. Lorsque Patočka publie sa thèse d'habilitation en 1936, non seulement il est influencé par Edmund Husserl sur un plan théorique qui, au même moment, élabore et expose La Crise des sciences européennes et la phénoménologie transcendantale (1954) ${ }^{18}$, mais aussi parce qu'il participe lui-même à un mouvement de défense de la philosophie européenne à Prague, au moment où l'avenir de l'Europe se joue. En 1933, Husserl, de par son origine juive et les nouvelles lois antisémites, se voit interdit l'accès à l'université allemande de Fribourg-en-Brisgau. Radié du corps professoral en 1936, il refuse de partir aux ÉtatsUnis, où l'Université de Californie lui ouvre ses portes, préférant rester au cœur de la «crise » en Europe. Il donne alors des conférences en Autriche et dans la République tchécoslovaque, pays n'étant pas encore tombés sous le joug de l'Allemagne nazie. Là, le Cercle philosophique de Prague ("Filozofický Kroužek»), fondé par Emil Utitz et Jan Blahoslav Kozák en 1934, se donne pour mission de défendre les "idéaux d'humanité " dans l'esprit de Tomáš Garrigue Masaryk et de donner asile à la phénoménologie alors interdite en Allemagne. Après la conférence de Husserl, le 7 mai 1935 au Kulturbund à Vienne, laquelle s'intitule "La crise de l'humanité européenne et la philosophie ", Patočka, le secrétaire du Cercle, l'invite au sein des deux universités de Prague, dont l'une est allemande. De par le succès important de ces dernières conférences, alors qu'il avait renoncé à écrire un nouveau résumé et une introduction systématique à la phénoménologie, Husserl rédige la Krisis, qui va constituer son dernier ouvrage. Celuici,-arrive évidemment au moment où la seconde Guerre Mondiale apparaît :

Ironie du sort, c'est à la veille de la seconde conflagration mondiale, destinée à éliminer définitivement l'Europe de la direction du monde, que Husserl écrit l'ouvrage qui contient sa conception phénoménologique de l'histoire. Il est vrai que la guerre fait en même temps de la science et de la technique européenne un trait d'union planétaire. Trait d'union, la civilisation européenne le devient cependant dans la version dont La crise des sciences européennes signale le caractère de déclin, montrant qu'il s'y est produit une perte de sens, la perte de l'idée téléologique donatrice de sens qui constitue, selon Husserl, l'essence intime, spirituelle de l'Europe ${ }^{19}$.

Aussi, comme l'explique Ludwig Landgrebe dans la Préface de Le Monde naturel, lorsque Husserl quitte Prague en 1935, ce dernier pousse Patočka à soutenir au plus vite sa thèse d'habilitation, ce qui est aussi l'avis de son directeur de thèse Kozák. En effet, si la situation de l'Europe est préoccupante et qu'il faille dénoncer la "crise » dans son origine et ses fondements, le concept de "monde naturel» marque d'abord une rupture avec le dogmatisme des sciences dont le positivisme doit être dépassé, non seulement pour des raisons théoriques, mais parce qu'il est néfaste à la vie, c'est-à-dire 
l'activité de l'homme ordinaire. Ainsi, dans sa volonté de réunification du sujet naïf soumis aux diktats des sciences et du monde, ressort un véritable engagement de la part de Patočka qui se pose comme un combattant face à la normalisation de la politique tchèque et de la montée du communisme des années 1930. Ayant lui-même fait l'expérience du front, ses activités sont interrompues à l'Université Charles par l'invasion allemande en 1939, elles reprennent juste après la seconde guerre mondiale et sont de nouveau perturbées en 1948. Il enseigne de nouveau vers 1968, mais juste avant sa retraite. Aussi, c'est dans "un minuscule appartement en sous-sol» que Patočka rédige ses travaux; là, il organise "un séminaire de phénoménologie privé, pratiquement clan-destin $»^{20}$. Lorsque son activité intellectuelle s'arrête tragiquement, le 13 mars 1977, suite à une hémorragie cérébrale, il vient d'essuyer maints interrogatoires et persécutions administratives menés par la police tchèque. Il était devenu le porte-parole du groupe des Droits et de l'homme et du citoyen pour la Charte $77^{21}$.

10 Ainsi, il existe un rapport très étroit entre la vie du philosophe tchèque lui-même, la force d'un contexte de crise dont il n'est pas la cause, et l'élaboration d'une pensée théorique qui s'appuie d'abord sur la phénoménologie. Lorsque Patočka pointe la crise spirituelle des années trente, dans la continuité des idées de Husserl qui se trouvent dans la Krisis, différentes "conceptions du monde " apparaissent sous la forme d'une double histoire des idées. Dans Le monde naturel comme problème philosophique (1936), le Chapitre II propose une succession de conceptions liées à la subjectivité qui débute avec Descartes, continue avec Kant, Fichte, Schelling, Hegel, et se termine avec Husserl. Dans le supplément à la deuxième édition tchèque, Le monde naturel dans la méditation de son auteur trente-trois ans après (1970), la même chronologie apparaît avec des ajouts, partant encore de Descartes, puis citant Spinoza, Leibniz, Locke, Kant, Hegel, Feuerbach, Mach, Avenarius, Brentano, Uphues, Schwarz, Bergson, Husserl, et enfin Heidegger. Dans ce sens, non seulement Patočka applique une généalogie à la phénoménologie, afin de comprendre son origine et ses fondements, mais il essaie de saisir le mouvement spirituel global ayant mené à la conception d'un monde en crise au sein de la modernité. Ce qu'il faut noter là, au-delà de l'effort chronologique et de l'addition simple d'auteurs, c'est que la compréhension que Patočka fait du «monde naturel » devient plus réaliste, liée à la vie humaine concrète dans la communauté et l'histoire. Des auteurs comme Ernst Mach ou Richard Avenarius fondent en effet l'empiriocriticisme dont le projet vise précisément la fondation d'une «conception naturelle du monde » reposant sur l'expérience pure. Franz Brentano ou Goswin Karl Uphues s'intéressent eux à une psychologie empirique. Tomáš Garrigue Masaryk, que Patočka soutiendra toute sa vie, est le fondateur du Parti dit « réaliste » en 1900. Notons enfin l'influence du pédagogue Coménius (1592-1670) que Patočka reprend dans ses Conférences de Louvain sur la contribution de la Bohême à l'idéal de la science moderne (1965) qui milite pour une réforme de l'éducation afin de sortir de la guerre de Trente Ans ${ }^{22}$. Ce réalisme, qui montre que Patočka a le sentiment lui-même de s'engager dans un monde qui décline, a par la suite une incidence importante sur la formalisation de sa pensée qui cherche à limiter le caractère purement subjectif de l'homme devant les événements concrets de la vie. 


\section{La force du monde et son origine polémique}

11 Afin de comprendre le "monde naturel », Patočka ne se limite pas à une histoire des idées sur un plan théorique, mais va chercher dans l'histoire concrète les déterminants sociaux qui orientent ses différentes conceptions. Ainsi, au-delà d'une permanence dans l'ébranlement du monde qui s'exprime sous la forme du sentiment du déclin chez le sujet, des crises sociales apparaissent et donnent plus ou moins d'amplitude à la vie, car elles sont très concrètes. Cette concrétude est ambivalente sur un plan historique car, sous-couvert d'une conception du monde moderne qui apparaît sous le jour du rationalisme dogmatique, elle n'en demeure pas moins réelle et efficiente dans son application à l'expérience humaine. Ainsi, il faut bien distinguer la conception du monde en tant que cause de l'histoire et sa représentation a posteriori qui est l'élaboration du penseur en tant qu'il pense l'histoire à partir de ses effets dans le réel.

Dans " Les guerres du $\mathrm{XX}^{\mathrm{e}}$ siècle et le $\mathrm{XX}^{\mathrm{e}}$ siècle en tant que guerre ", Patočka trouve une accroche permettant de relier l'histoire des vécus du sujet d'expérience (Geschichte) à celle des événements du monde extérieur (Historie) ${ }^{23}$. En effet, si la guerre constitue un " grand » événement, c'est en raison de la «force » qu'elle applique aux sujets qui non seulement résonne avec leur existence personnelle mais peut s'exprimer dans la communauté sous la forme du discours, de la pensée et de l'opposition. Dès le début du texte, Patočka critique les approches historiques traditionnelles qui n'expliquent la guerre qu'à travers les "idées de la paix, du jour et de ses intérêts " ${ }^{24}$. Dans ce sens, la guerre en tant que telle n'a pas de vertu explicative et l'obscurité des hommes - la mort, la finitude de l'être, la peur, l'angoisse - est toujours perçue de façon négative, comme la conséquence triste et dommageable de raisons supérieures, politiques ou sociales, animées par l'esprit du bien. Et pourtant, après la victoire, force est de constater que toujours, des nouveaux conflits apparaissent. Sont-ils pour autant animés par l'idée du Bien? Plutôt que de comprendre le conflit à partir de l'étude des forces opposées, Patočka opte pour une approche réaliste qui comprend les deux camps comme animés par un même mouvement, "résultat de l'objectivation violente du subjectivisme excessif des Temps modernes $»^{25}$. Dans ce sens, c'est d'abord l'esprit de l'Europe qui est en crise dont la concentration des forces va entrainer un surplus et une explosion. Si la Krisis de Husserl a permis de mettre à jour le «danger » (Die Gefahr) du positivisme, moment encore théorique qui part d'une critique de la mathématisation galiléenne qui a vidée la science de son sens à partir d'une technicisation des savoirs ${ }^{26}$, c'est surtout chez Heidegger que Patočka puise sa réflexion historique à partir d'une critique de la technique moderne et de l'entente de la finitude de l'existence. Dans ce sens, non seulement, la vie de l'homme s'est dénaturée, parce qu'elle est devenue l'objet des sciences, mais aussi parce que son étude, est devenue une technique qui voile son sens originel dans l'illusion de son exploitation infinie. Aussi, parce que différentes puissances sont engagées dans un même dispositif technique " surcivilisationnel $»^{27}$ qui consiste à exploiter la nature ordinaire sans regarder la limite des ressources du monde, non seulement des conflits territoriaux apparaissent, mais aussi des guerres dont le but est d'appliquer à l'homme la même force technique afin qu'il puisse gagner de l'espace, jusqu'à sa fin: "Pourquoi la transformation énergétique du monde ne peut-elle se faire que par voie de guerre? Parce que la guerre, l'opposition aigüe, est le moyen le plus efficace de libérer les forces accumulées? $»^{28}$. 
Inspiré par Teilhard de Chardin, Patočka est plus matérialiste qu'Heidegger, car l'homme conquiert sa liberté au « front » tout en subissant la force du monde ${ }^{29}$. Ce front est une ligne de démarcation dont le mouvement mécanique manifeste l'aliénation de l'homme et sa technicisation dans la recherche d'une territorialité plus grande où l'esprit humain a quasiment disparu. Cette matérialité, bien que minorée par Heidegger sur un plan ontologique, est importante car elle marque potentiellement la destruction réelle de l'humanité et donc la fin définitive du monde. En effet, «le dévoilement qui régit la technique moderne est une pro-vocation (Herausfordern) par laquelle la nature est mise en demeure de livrer une énergie qui puisse comme telle être extraite (herausgefördet) et accumulée $»^{30}$. Dans ce «requérir " qui provoque la nature, réside bien un danger qui non seulement implique l'homme mais le comprend, le sommant lui-même à fournir une énergie renouvelable, alors qu'il est concrètement fini. Se trouve donc en suspend l'appel de la destruction dans l'incapacité de la nature à fournir une ressource infinie dont l'homme fait pourtant partie, en d'autres termes l'inévitable sacrifice de l'homme devant une nature qu'il ne peut pas contrôler et dont il a épuisé le fonds. Aussi, si Heidegger établit une rupture au lendemain de la seconde guerre mondiale, lorsqu'il questionne non plus la technique mais la « technique moderne » en rapport notamment à la bombe atomique, il n'y voit en rien une fatalité, mais un "appel libérateur» dans l'ouverture de l'homme à l'essence de la technique. À l'inverse, chez Patočka, parce que les effets de la technique moderne sont bien réels, ils peuvent faire disparaitre l'homme et la planète toute entière. Alors, si l'appel de l'essence de la technique est un espoir pouvant permettre à l'homme de sortir de la crise, sa concrétude peut aujourd'hui, et à la différence d'avant, faire disparaître l'homme, et par voie de conséquence l'espoir total d'un monde meilleur.

Pour Patočka, il faut sacrifier l'idée d'un monde manichéen qui rechercherait dans la paix l'illusion d'un sauvetage. L'opposition du bien et du mal, qui s'instaure entre deux clans pendant une guerre, renoue avec l'opposition originelle montrée par Platon, laquelle dissocie un monde fait d'illusions et un autre qui serait idéal. Cette séparation (chorismos), si elle se veut problématique, se résout de façon dialectique à condition d'être reprise dans la temporalité de l'être, ou encore le mouvement de l'existence, dont le monde est dynamique. La perspective de l'histoire se vit ainsi au sein de l'existence humaine, parce que sa temporalité conserve le sens de la crise qui fluctue entre guerre et paix depuis l'origine du monde. Dans ce sens, il n'y a plus de guerre ou de paix, mais une guerre-paix telle qu'Héraclite l'affirmait. Selon lui : « la guerre est le père de toutes choses, et de toutes choses il est le roi ; c'est lui qui fait que certains sont des dieux et d'autres des hommes, que certains sont des esclaves quand d'autres sont

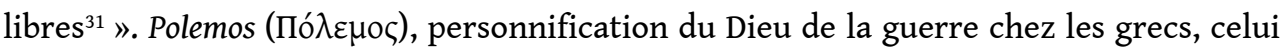
qui divise, est la force suprême, bien qu'invisible, qui équilibre le monde et le fait devenir. Les hommes ne sont hommes que parce que les dieux existent, qu'ils sont différents des hommes et s'opposent ; les dieux ne sont dieux que parce que les hommes existent, qu'ils sont différents des dieux et s'opposent. Et au sein même des hommes, les esclaves ne sont esclaves que parce que les hommes libres existent, qu'ils sont différents des esclaves et s'opposent ; les hommes libres existent que parce que les esclaves existent, qu'ils sont différents des hommes libres et s'opposent. Si Polemos est opposition, il est la résultante d'un équilibre entre des forces contraires dont l'expression finale est un phénomène d'harmonie où, « ce qui est différent de soi-même s'accorde avec soi-même; il y a une harmonie dans les deux directions, comme dans l'arc et dans la lyre ${ }^{32}$. Dans le cas de l'arc, c'est l'opposition parfaite entre le bras droit 
tendant la corde et le bras gauche maintenant l'arc qui aboutit à l'atteinte de la cible. Comme pour la lyre, la fin est un acte dont la beauté exprime la qualité de l'ajustement entre les forces en tension. Alors Polemos est arkhè, c'est-à-dire le mouvement à l'origine de toutes les choses qui s'exprime de façon harmonieuse dans l'opposition des forces existant dans le réel. En d'autres termes, l'aspect polémique du monde est le fond ontologique duquel adviennent les événements qui s'abattent sur l'homme et résonnent avec son existence ; et, face à sa force, l'homme peut soit se sacrifier, c'est-àdire disparaître, ou bien réagir.

\section{Le sacrifice du sujet}

Sur les onze textes qui constituent l'ouvrage Liberté et sacrifice, le sacrifice est précisément conceptualisé dans le texte «Les périls de l'orientation de la science vers la technique selon Husserl et l'essence de la technique en tant que péril selon Heidegger ", qui correspond à la conférence prononcée par Patočka au Congrès philosophique de Varna en $1973^{33}$. Dans ce texte, si l'auteur tchèque continue de puiser dans les réflexions à la fois de Husserl et de Heidegger, il prend non seulement un ton polémique vis-à-vis de ses deux prédécesseurs, mais fait preuve d'activisme puisqu'il érige le sacrifice en action radicale, laquelle caractérise l'essence de la compréhension véritable qui porte sur l'homme et le monde au sein duquel l'auteur vit: «L'idée du sacrifice est d'origine mythico-religieuse. [...] L'idée paradoxale, c'est qu'on gagne en consentant une perte volontaire ${ }^{34} »$. En effet, se sacrifie la personne qui croit que devant l'autre il y a quelque chose à gagner, quand bien même il doit périr. Cela résulte de la perception d'une différence subjective, laquelle est jugée souvent au nom d'un transcendantal, qui efface les contraintes du monde réel. Aussi, parce que le sacrifice manifeste un effacement de soi qui se traduit par la blessure, la souffrance, la torture, la mort, son acte est toujours un choc intersubjectif qui fait apparaître chez l'autre, quelque chose d'étrange, voire de contre-nature. Cet étonnement, qui naît du sacrifice, force le sujet à percevoir le monde car sa violence est insupportable et rappelle la finitude de l'existence - la possible disparition de soi. Ce sentiment peut éveiller deux sentiments contradictoires: soit la peur du sujet qui devant l'acte horrible désire s'isoler, se cacher, disparaître du monde naturel ou s'enfuir ; soit la reconnaissance du courage, et de la beauté d'un geste qui s'attache à un idéal, ralliant l'autre à un même combat au sein du front solidaire. Une confusion demeure. Il y a d'abord le sacrifice de l'homme au sein du monde naturel qui est chosique et participe d'une précompréhension historique de la vie, c'est-à-dire qui ne pense pas vraiment, ou alors réfléchit seulement sous le joug de la ratiocination et se pose en victime du fond technico-scientifique; et puis, il y a le sacrifice qui est repris dans son aspect polémique, c'est-à-dire en tant qu'il exprime la négation du monde, dont le fond " naïf », qu'il soit mythico-religieux ou technique, fait l'objet d'une compréhension au sein de l'existence humaine. Cette compréhension, elle n'est pas rationnelle car elle marque une conversion (metanoia) réelle du sujet qui n'est plus passif devant le monde, mais affirme son opposition et dit «non" à ce qui n'est pas posé comme un questionnement véritable. Le sacrifice est ainsi une réponse à la finitude de la vie en ce qu'il donne sens à l'histoire à partir de la description de la disparition progressive de l'homme au sein du monde naturel. Il s'agit alors d'un «sacrifice radical » où le sujet prend la mesure de sa responsabilité qui est une réponse négative devant le monde qui se situe en crise. Si l'histoire vise à donner sens aux crises qui apparaissent sous la 
forme d'événements, pas seulement des événements individuels mais ceux intersubjectifs qui touchent à la société ou au monde de l'être-avec (Mitsein), alors il faut sacrifier l'attitude du monde naturel qui consiste à donner sens hâtivement aux choses, au profit d'un habitus problématique dont la négation peut et doit être partagée. Dès lors, non seulement le concept de «monde naturel » est sacrifié sur un plan théorique, mais il est converti en monde historique qui se trouve être la totalité de tous les étants et dont la saisie est possible en partie dans la compréhension événementielle des crises et des guerres.

Enfin, l'engagement du penseur comme sacrifice selon Patočka demeure ambigu car, s'il critique autant le subjectivisme de Husserl que le manque de concrétude chez Heidegger, lui, les dépasse-t-il vraiment? Il est clair que la vie de Patočka montre un investissement dans le monde et des actions qui témoignent de sa réflexion dans la recherche d'un sens et de ses applications au sein du réel. S'il faut plutôt valoriser l'aspect discursif de son travail, et en ce sens, parler d'une vie de l'esprit qui s'opposerait à une autre moins abstraite, cela nous semble contradictoire au regard de la pensée de l'auteur même qui fait du langage l'« instrument de notre praxis, servant à l'échange intersubjectif de contenus spirituels [mais aussi] d'ores et déjà, une saisie de la réalité $»^{35}$. Aussi, toute critique, parce qu'elle advient « dans » le monde et manifeste son existence (que la forme de l'esprit représente peut-être le mieux), est d'abord constituée par le monde qui lui a imposé des événements. Elle donne ainsi au monde un sens qui participe, non pas seulement à son mouvement, mais à son changement qui est bien réel, puisqu'il exprime les préoccupations du sujet dans l'histoire, s'incarnant dans des œuvres qui, au-delà des vies individuelles, perdurent et font office de mémoire. Ces œuvres (par exemples des livres) participent à l'histoire comme elles imprègnent le monde naturel et offrent autant de possibilités aux autres hommes de trouver un sens problématique à la vie, bien qu'elles reposent sur des interprétations personnelles. Elles entraînent alors : "toute la vie individuelle et collective dans le domaine d'une transformation du sens, dans un domaine où la vie se voit obligée de changer entièrement de structure en changeant de sens. L'histoire n'est pas autre chose $»^{36}$.

17 Ce qu'il faut donc retenir, c'est la constitution de l'événement historique en rapport à l'autre, non plus comprise dans son rapport intersubjectif où le fond demeure un étant isolé, mais selon l'esprit et la vie de la communauté qui évidemment demeure insaisissable par un seul sujet. Dans ce sens, lorsque Patočka parle de «solidarité des ébranlés $»^{37}$, pour qualifier la compréhension de ceux qui savent que le monde est en crise et qu'il faut problématiser son sens afin de le rendre positif dans la réaction du sujet devant l'aliénation de soi et le déclin du monde, il confond peut-être une solidarité véritable et la tentative d'universalisation de l'esprit qui cherche à unifier les conceptions du monde sous le concept de l'Un (ce qui correspond à l'Être, chez Heidegger). S'il est donc possible de distinguer sur un plan théorique le monde naturel, du monde de la pensée qui le comprend et donne du sens à l'histoire, ce dernier est ici posé par Patočka comme supérieur. Cette supériorité manifeste une sortie de l'esclavage du monde naturel qui correspond à l'attitude de l'homme naiff, pris dans le flux de l'activité ordinaire, soumis à la rationalisation technique et aux conflits sociaux, mais aussi à une élévation de l'esprit qui permet à l'homme de devenir authentique, c'est-à-dire de saisir le sens réel de ce qu'il est dans le monde à une période donnée de l'histoire. La description de cet Être, qui est en fait le fonds de l'histoire, n'apparaît pas comme telle chez Patočka, car il néglige l'activisme de son œuvre. En effet, même dans 
les Essais hérétiques, Patočka limite l'activité de l'homme ordinaire à sa passivité devant la force du monde et ne reprend pas le mouvement unifiant de l'esprit dans, non plus son mouvement concret, mais l'orientation de l'action qui succède à la saisie du monde vécue comme un problème. Il est vrai que l'attitude de l'esprit peut montrer le "non » face au monde, s'opposer aux effets réels qui sont des atteintes à la liberté humaine, faire du phénomène un événement a-subjectif, plus loin même se sacrifier dans la recherche d'un sens oublié ou déclinant, sans que le penseur n'ait la garantie de trouver un terrain pour faire croître ses idées. Pour autant, est-il sûr que ce mouvement de l'esprit soit plus efficient que des actions non-discursives, celles qui se privent de mots et recherchent des effets concrets dans le monde en aidant directement à agir les autres? Dès lors, dans la concrétude de l'esprit qui prend pour objet le monde social et s'oppose aux restrictions réelles, il y a bien un sacrifice de soi dont les effets discursifs sont un appel au front commun, mais qui au fond, demeure personnel et naï ${ }^{38}$. Alors, pour que les hommes deviennent solidaires, il faut peut-être dépasser l'expression de la pensée a-subjective qui s'incarne dans le discours seul quand bien même il exprime l'ébranlement de l'existence éprouvé par les autres. Tel un appel à l'Être, la pensée de Patočka s'abîme finalement avant l'heure dans une ontologie réaliste qui, dans son sacrifice du sujet, a fait comme s'il pouvait complètement disparaître ${ }^{39}$.

\section{NOTES}

1. La thèse d'habilitation de J. Patočka Le Monde naturel comme problème philosophique, est traduite de façon assez confidentielle par J. Daněk et H. Declève en 1976. Depuis 1981, avec Les essais hérétiques sur la philosophie de l'histoire (préface de P. Ricœur); c'est E. Abrams qui traduit essentiellement ses ouvrages.

2. Plus formellement: "la nouvelle figure de la phénoménologie, dans toutes ses versions, procède d'un changement de paradigme, c'est-à-dire de la modification du concept de phénomène désormais entendu comme événement (Ereignis) », C. Sommer, «Transformations de la phénoménologie » in Revue Sciences/Lettres [En ligne], URL : http://journals.openedition.org/ rsl/235). Comme l'explique C. Sommer, selon «Gondek et Tengelyi, la génération actuelle radicalise et systématise donc les percées de ses aînés au-delà de Husserl et Heidegger - ajoutons plus prudemment : au-delà d'un certain Husserl et d'un certain Heidegger - en opérant une transformation $\mathrm{du}$ concept même de phénomène. Le phénomène excède l'objectivité husserlienne (non-objectif, il déborde l'horizon intentionnel), et l'étantité heideggérienne (nonétant, il se situe dans l'“autrement qu'être”) » (Ibid., §9). L'ouvrage de H.-D. Gondek et L. Tengelyi cité est Neue Phänomenologie in Frankreich (Berlin, Suhrkamp, 2011). Il est un état des lieux important sur l'état de la phénoménologie actuelle en France, lequel poursuit la réflexion entamée par Bernhard Waldenfels (Phänomenologie in Frankreich, Francfort-sur-le-Main, Suhrkamp, 1983) et la troisième partie du second volume de Herbert Spiegelberg ("The French Phase of the Movement », in The Phenomenological Movement : A Historical Introduction, 2 vol., La Haye, Nijhoff, $3^{\text {e }}$ édition, 1982 [1960], p. 395-592).

3. É. Tardivel, La liberté au principe. Essai sur la philosophie de Patočka, Paris, Vrin, Préface de Françoise Dastur, 2011, p. 8-9. 
4. Sur la phénoménologie «a-subjective » de J. Patočka, nous renvoyons à l'article de D. Duicu "La phénoménologie asubjective de Jan Patočka, une phénoménologie non intentionnelle », in Bulletin d'analyse phénoménologique VI 8, 2010 (Actes 3), p. 230-243, ISSN 1782-2041 http:// popups.ulg.ac.be/bap.htm.

5. Suivant le romantisme du Lebenswelt, la Weltanschauung fait référence à une vision (Anschauung) du monde (Welt) que l'on trouve notamment chez Hölderlin ou Novalis, mais aussi Fichte, Kant, Hegel ou Heidegger. Aussi, s'il existe des conceptions du monde, celles-ci sont déterminables sur un plan historique et correspondent à des périodes. "Quand nous méditons l'essence des Temps Modernes, nous posons la question de la "conception moderne du monde" (neuzeitliches Weltbild). Et nous caractérisons alors cette "conception du monde" en la distinguant de la "conception médiévale du monde" et de la "conception antique du monde". Mais pour quelle raison nous enquérons-nous d'une "conception $\mathrm{du}$ monde" lorsque nous tentons d'interpréter une époque ? Chaque époque de l'histoire a-t-elle donc "sa conception du monde", et cela de telle sorte qu'elle se préoccuperait toujours déjà de "conception du monde" ? Ou bien ne serait-ce pas exclusivement une façon moderne de se représenter les choses que de s'enquérir de la "conception du monde" ?» (M. Heidegger, «Die Zeit des Weltbildes », in Holzwege, Ga 5, p. 88 ; trad. par W. Brokmeier, "L'époque des "conceptions du monde" ", in Chemins qui ne mènent nulle part, Paris, Gallimard, Coll. Classiques de la philosophie, 1962 [1934-1946], p. 116).

6. J. Patočka, Essais hérétiques sur la philosophie de l'histoire (dorénavant EH), Lagrasse, Verdier, trad. par E. Abrams, 1999 [1975], p. 194.

7. En italique dans le texte. J. Patočka, Le monde naturel comme problème philosophique (dorénavant MN), Paris, Vrin, trad. par E. Abrams, 2016 [1936], p. 32.

8. J. Patočka, Liberté et sacrifice. Écrits politiques (dorénavant LS), Grenoble, Jérôme Millon, trad. par E. Abrams, 1990 [1934-1976], p. 16.

9. Concept que Patočka reprend à « Weischedel [...] ou, pour parler le langage de Heidegger, le non-manifeste comme fondement de toute ouverture et de toute manifestation » (EH, p. 127).

10. J. Patočka, Platon et l'Europe, Séminaire privé du semestre d'été 1973 (dorénavant PE), Lagrasse, Verdier, trad. par E. Abrams, 1983 [1973], p. 50.

11. Ibid., p. 10-11.

12. MN, p. 96.

13. PE, p. 52.

14. Du grec $\chi \omega \rho$ plouó chōrismós, « séparation » chez Parménide et surtout chez Platon en rapport aux données intelligibles ou sensibles.

15. LS, p. 251.

16. Il existe beaucoup trop de commentaires autour de la pensée de Platon permettant d'expliquer la vocation politique du philosophe. Retenons simplement que Patočka emploie la pensée du philosophe grec parce qu'il voit une ressemblance entre la crise historique que ce dernier traverse pendant l'Antiquité et celle du début du $\mathrm{XX}^{\mathrm{e}}$ siècle, lesquelles expliquent la naissance de la discipline philosophique, et deux mille ans après, celle de la phénoménologie. Voir notamment le séminaire privé donné par Patočka en 1973 ayant donné lieu à l'ouvrage Platon et l'Europe.

17. Voir La crise du sens (1977) où se trouve une bibliographie très détaillée permettant d'apercevoir l'étendue du propos de J. Patočka (J. Patočka, La crise du sens, Tome 1, Comte, Masaryk, Husserl, et Tome 2, Masaryk et l'action, Bruxelles, Éditions OUSIA, 1985 et 1986 [1977]).

18. Ouvrage inachevé, La Crise des sciences européennes et la phénoménologie transcendantale correspond chez E. Husserl au volume VI des Husserliana : Die Krisis der europäischen Wissenshaften und die transzendentale Phänomenologie (dorénavant Krisis). Il est seulement publié à titre posthume en 1954 chez Martinus Nijhoff. Pour la traduction française, nous faisons référence à Edmund Husserl, La Crise des sciences européennes et la phénoménologie transcendantale, Paris, Gallimard, Coll. nrf, trad. par G. Granet, 1976 [1954]. 
19. EH, p. 84.

20. R. Jakobson, Le curriculum vitce d'un philosophe tchèque, 1977, in EH, p. 243.

21. Alors que le gouvernement communiste tchèque, mené par Gustáv Husák, s'est engagé à respecter les Droits de l'homme lors de la Conférence d'Helsinki en 1975, la Charte 77 (Charta 77 en tchèque) est une pétition qui le rappelle à l'ordre, devenu le vassal loyal de la Russie dans le processus de normalisation, source d'oppressions.

22. C'est d'abord l'aspect réformiste de Coménius qui plaît à Patočka. Sur un plan historique, il faut remarquer que le mouvement de la Renaissance nationale tchèque (České národní obrození), qui est particulièrement saillant au XVIII ${ }^{\mathrm{e}}$ et $\mathrm{XIX}^{\mathrm{e}}$ siècle, milite pour un retour à l'esprit de l'Antiquité, qui paraît beaucoup plus réaliste que celui du Moyen-âge rempli de dogmes et de croyances.

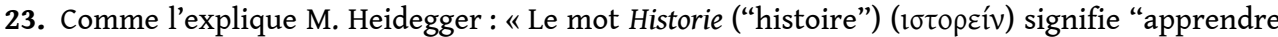
en s'informant et rendre visible" et désigne ainsi un mode de représentation. Au contraire le mot Geschichte (histoire) signifie ce qui arrive, pour autant qu'il est préparé et commis de telle et telle manière, c'est-à-dire mis en ordre et envoyé » (M. Heidegger, " Wissenschaft und Besinnung ", in Vorträge und Aufsätze, Gesamtausgabe, vol. 7, Francfort-sur-le-Main, Klostermann, 2000, p. 58 ; trad. par A. Préau, «Science et médiation », in Essais et conférences, Paris, Gallimard, Coll. Tel, 1980 [1958], p.71).

24. EH, p. 191.

25. Ibid, p. 190.

26. Krisis, p. 53.

27. « La surcivilisation et son conflit interne », in LS, p. 99-178.

28. EH, p. 197.

29. Ibid, p. 198.

30. M. Heidegger, « Die Frage nach der Technik », in Vorträge und Aufsätze, Gesamtausgabe, vol. 7, Francfort-sur-le-Main, Klostermann, 2000, p. 15 ; trad. « La question de la technique », in Essais et conférences, Paris, Gallimard, 1958, p. 20.

31. Héraclite, Fragments [Citations et témoignages], Paris, GF Flammarion, $2^{\mathrm{e}}$ éd. corrigée, trad. par J.-F. Pradeau, 2004 [2002], p. 126.

32. Ibid., p. 151.

33. «Die Gefahren der Technisierung in der Wissenschaft bei E. Husserl und das Wesen der Technik als Gefahr bei M. Heidegger ». Conférence prononcée au Congrès mondial de philosophie en septembre 1973 à Varna. Une autre version existe dans les Cahiers de philosophie, $\mathrm{n}^{\circ}$ 11, janvier 1991. Aux onze textes, il faut rajouter en annexe une réflexion au sujet de l'interview donnée par M. Heidegger au Spiegel intitulée « Réponses et questions sur l'histoire de la politique ».

34. LS, p. 271.

35. MN, p. 130.

36. EH, p. 108-109.

37. Ibid, p. 213.

38. Patočka parle « d'expérience individuelle » et « d'isolement », Ibid, p. 212.

39. N'est-ce pas là la critique qu'on pourrait faire par ailleurs à Heidegger qui, dans son tournant (Kehre), montre une pensée qui s'éloigne du Dasein, voire même l'élimine, sans que cela ne soit véritablement possible, puisqu'il reste le penseur? 